\title{
Research on Routing Algorithm of Wireless Sensor Networks Based on Energy Balance
}

\author{
He Qian \\ Chongqing College of Electronic Engineering, Chongqing 401331, China \\ 11047225@qq.com
}

Keywords: Routing Algorithm, Wireless Sensor Networks, Energy Balance

\begin{abstract}
In order to improve the lifetime of wireless sensor networks, solve the problem of energy imbalance in LEACH, an energy balanced wireless sensor network node routing algorithm is proposed. Firstly, the monitoring area is regarded as a fan-shaped area centered on the base station, and the fan-shaped area is divided into arc-shaped blocks with different sizes. The nodes in each arc-shaped block form a cluster. Cluster heads are generated according to the residual energy of nodes, Jump and multi-hop combination of inter-cluster communication mechanism, and finally, simulation experiments are conducted to test the performance of the algorithm. The results show that the proposed algorithm can effectively improve the energy utilization rate of the network, balance the energy consumption of the nodes, prolong the network lifetime of the wireless sensor, which is suitable for the applications with high requirements on the network life cycle.
\end{abstract}

\section{Introduction}

With the rapid development and maturing of communication technology, embedded computing technology and sensor technology, miniature sensors with the ability of sensing, computing and communication begin to appear in the world. The wireless sensor networks formed by these miniature sensors have aroused people's great attention. Wireless sensor network integrates sensor technology, embedded computing technology, distributed information processing technology and communication technology, which can monitor, perceive and collect the information of various environments or monitoring objects in the network distribution area in real time, Processing, access to detailed and accurate information, sent to the needs of these users. The sensor network allows people to get a lot of detailed and reliable information anytime, anywhere and any environmental conditions. Therefore, such a network system can be widely applied in fields such as national defense, national security, environmental monitoring, traffic management, medical and health care, manufacturing, and fighting against terrorism. Wireless sensor networks are receiving more and more attention, and more and more researchers are involved in the research of wireless sensor networks.

Often, wireless sensor networks consist of hundreds of nodes deployed on a large scale and have different design goals than traditional wireless networks such as WLANs and cellular mobile phone networks, which are optimized for routing in high-speed, mobile environments and resource management strategies to maximize the utilization of bandwidth, while providing users with a certain quality of service assurance. In wireless sensor networks, most nodes are stationary, except for the few nodes that need to be moved. Because they often run in harsh or even dangerous remote environments that are inaccessible to people and energy can not be replenished, designing effective strategies to extend the life cycle of a network becomes a central issue in wireless sensor networks.

At the beginning of the research of wireless sensor network, people once thought that the mature Internet technology combined with Ad-hoc routing mechanism was sufficient for the design of sensor networks. However, in-depth studies show that the sensor networks have distinctly different technical requirements from traditional networks. The former is data-centric, the latter for the purpose of data transmission. In order to adapt to a wide range of applications, the traditional network design follows the "end-to-end" edge theory, emphasizing that all function-related 
processes are placed on the network end system. The intermediate nodes are only responsible for forwarding data packets. Sensor network, this may not be a reasonable choice. Some protocols and algorithms designed for ad hoc networks that are self-organizing may not be suitable for the characteristics and applications of sensor networks. The role of node identification (such as address, etc.) in the sensor network is not very important, because the application is not very concerned about the information on a single node, the application-specific intermediate nodes on the data processing, integration and caching is also very necessary. In dense sensor networks, the distance between adjacent nodes is very short, and the low-power multi-hop communication mode saves power while increasing the covert communication and avoiding the long-distance wireless communication susceptible to external noise Impact. These unique requirements and constraints put forward new technical problems for the research of sensor networks.

\section{The Concept of Wireless Sensor Networks}

Wireless sensor network is a multi-hop self-organizing network system formed by a large number of inexpensive micro-sensor nodes deployed in the monitoring area through wireless communication. Its purpose is to cooperatively perceive, collect and process network coverage in the geographical area Perception of the object's information, and sent to the observer.

Sensors, objects of perception and observers are three basic elements of a wireless sensor network. Wired or wireless networks are the means of communication between sensors, sensors and observers, for establishing a communication path between the sensor and the observer, for cooperation Perception, acquisition, processing, release of the perception of information is the basic function of the sensor network. Some or all of the nodes in a wireless sensor network can move. Therefore, the topology of a wireless sensor network also dynamically changes as nodes move. Ad-hoc communication between nodes, each node can act as a router, and each node has the ability to dynamically search, locate and restore the connection.

The sensor node is usually a miniature embedded system, its processing power and communication capacity is relatively limited, by carrying a battery with limited power supply. The node consists of sensor module, processor module, wireless communication module and energy supply module. The sensor module is responsible for monitoring the collection and data conversion of the information in the area; the processor module is responsible for controlling the operation of the entire sensor node, storing and processing data collected by itself and data sent by other nodes; and the wireless communication module is responsible for performing wireless communication with other sensor nodes, Exchange control messages and send and receive data; energy supply module for sensor nodes to provide the necessary energy to run, usually micro-battery.

\section{The Wireless Sensor Network Energy Balance Consumption Routing Protocol}

SPINSPIN (Sensor Protocol for Information via Negotiation) Assuming that each node in the network is a base station, the information collected by each node is dispersed throughout the network, so that each node in the network can have all of the network Data information. At this moment, if the user wants to inquire the data and only needs to communicate with any node in the network. The crux of the problem at this time is how nodes effectively disseminate the collected information to the network while maintaining a small amount of traffic. SPIN uses data negotiation to reduce the amount of data disseminated traffic. Assuming that adjacent nodes in the network have a large amount of similar information, nodes only need to exchange data that no one node has. For example, when a node collects new data, the node broadcasts a description of the new information it possesses to the network. If other nodes are interested in this information, the node with the new information is required to transmit data to it. After this data negotiation process, the actual data exchange is started. SPIN requires upper-level protocols to use a mechanism that accurately describes the raw data. The exchange of any raw data uses data descriptions for data negotiation to determine if data exchange is required. Such a mechanism guarantees the minimum transmission of redundant data in the network. SPIN can also adjust the operation of the protocol according to its 
own resources, such as the amount of energy. SPIN is a time-driven routing that spreads out periodically, even when no data information is required.

SPIN improves the inefficiencies of flood routing by negotiating data and adjusting energy usage. SPIN improves routing algorithm performance in two ways: First, the description of the data transmitted over the network is more efficient than the direct transmission of data; and second, flooding routes repeatedly transmit different copies of the same data message in some areas of the network. SPIN does not guarantee that data is distributed to all nodes in the network. For example, when a node has new data, it broadcasts to the network that it has new data. Neighbor nodes around it may have the same data information, so the information broadcast is limited to a certain area. Another node that is far away from this node may be interested in this one node but can not receive the information broadcast from the source node.

\section{The Data Collection Sensor Network Energy Balance Routing Protocol}

An important application of wireless sensor networks is monitoring applications, such as cropland crop monitoring, building health monitoring, lake water quality monitoring. In this application, the nodes need to periodically sense the monitored objects at regular intervals and send the collected data to the base station in a multi-hop manner. This type of sensor network has three types of features: centralized data collection, multi-hop data transmission and many-to-one traffic pattern, which are called data collection sensor networks.

Data Collection Sensor Network The traditional data collection mode is based on the station being stationary. Therefore, the nodes around the base station will bear all the loads in the network as the bottleneck of network performance, not only reducing the network throughput, Rapid death, thereby reducing the life of the network. However, the nodes in the network peripheral area far away from the base station need only send data collected by themselves or forward data of a few other nodes with less load. These nodes often have a large amount of residual energy at the end of the network lifetime. Therefore, how to balance the load of the neighboring nodes around the base station and realize the balanced energy consumption of the nodes and other nodes in the network is an important issue to be solved in the data collection sensor network. It can be considered that the balance of node load and node energy consumption balance belong to the same concept.

In order to balance the load on the nodes around the base station, there are several ways: First, deploy more nodes around the base station in turn, or deploy more energy nodes, this method can not fundamentally solve the problem of load balancing; the second, The introduction of mobile relay to share the load around the node around the base station, this approach can effectively extend the life of the network, but the load imbalance problem still exists; Third, by moving the base station to balance the load of these nodes, this method can be very A good balance of load, to achieve a balanced use of wireless sensor network node energy, but technically more difficult to achieve.

This paper first introduces a maximum and minimum energy path routing algorithm MCP, and then improves the algorithm for data collection sensor networks, and proposes a global energy-balanced routing algorithm GEBR. Compared with the MCP routing protocol, GEBR has established more candidate paths and combined the direct transmission and multi-hop transmission to reduce the imbalance of energy consumption in all areas, especially between nodes around the base station and other areas. Extend the life cycle of the network.

\section{The Balanced Energy Diffusion Routing Protocol}

Directed diffusion is a classic query-based planar routing protocol in wireless sensor networks. In directional diffusion, the sink node sends out the query task through the interest message, and floods the interest message to all the sensor nodes in the whole network or part of the region. The interest message is used to represent the task of querying and express information that the network user is interested in the monitoring area, such as environmental information such as temperature, humidity and light in the monitoring area. During the propagation of the interest message, the 
protocol establishes a reverse gradient of data transmission from the data source to the sink node on each sensor node hop by hop, and the sensor node transmits the collected data along a gradient direction to the convergence node. Directional diffusion routing mechanism can be divided into cyclical interest proliferation, data detection and path enhancement in three stages.

\subsection{Interest diffusion stage.}

During the stage of interest diffusion, sink nodes periodically broadcast interest messages to neighboring nodes. Interest message contains the task type, data transmission rate, time stamp and other parameters. These interests are probing nature, requiring nodes to send data at a lower rate. Each node caches the received interest and establishes a gradient relationship by recording the corresponding interested neighbors, data rate and timestamp. As interest spreads across the network, a data transfer gradient from the data source node to the sink node is established.

\subsection{Data detection phase.}

When the sensor node collects the data that matches the interest, the data is sent to the neighboring node on the gradient according to the data transmission rate on the gradient. Since it is possible to receive interest messages from multiple neighboring nodes, the node sends data to multiple neighboring nodes, and the sink node may receive the same data over multiple paths. After receiving the data forwarded by other nodes, the intermediate node first queries the interest list and discards the data if there is no matching interest. To avoid transmission loops, each interest corresponds to a data buffer pool, used to save the recently forwarded data. If there is a copy in the data buffer pool that matches the received data, the data is discarded.

\subsection{Path to strengthen the stage.}

After receiving these low-rate data, the sink node sends an enhanced message to the nearest neighbor of the data, and the enhanced message indicates that the sink node needs to send data at a high rate. Corresponding neighbor nodes in the same way, in turn deliver enhanced information to their neighbors until they reach the data source, which will build the data sent by the main path. The data is then sent to the sink node through the primary path.

\section{Conclusion}

Wireless sensor network is a multi-hop self-organized network system formed by a large number of inexpensive micro-sensor nodes deployed in the surveillance area and formed through wireless communication. It can be widely used in military, environmental monitoring and forecast, health care, smart home other fields. If the Internet constitutes a logical information world, changing the way of communication between people, then the wireless sensor network is the logic of the information world and the objective physical world together to change the interaction between mankind and nature.

\section{References}

[1] Xue Liang, Guan Xinping, Yuan Yazhou. Event-driven energy balance multi-flow aggregation routing algorithm in wireless sensor networks [J]. Control \& Decision. 201202

[2] Liang Jia-rong, Xu Xue-xin, Song Xiao-tian. An energy balance routing algorithm for wireless sensor networks [J]. Journal of Beijing University of Technology. 2012 (05)

[3] He Yunlu, Zhang Xin, Guo Hongyao, Zhang Wen. An energy balance routing game algorithm for wireless sensor networks [J]. Semiconductor Optoelectronics. 2014 (02)

[4] Liu Tao, Xie Chuhui. Energy Balanced Routing Algorithm for Wireless Sensor Networks [J]. Microelectronics and Computer. 2009 (10)

[5] Zhang Jinrong, Wang Yue, Wang Dong, Tang Xianlun. Clustering Routing and Topology 
Formation Algorithm for Combined Energy Distribute Round Robin Wireless Sensor Networks [J]. Instrumentation Technology and Sensor. 2009 (S1)

[6] Gao Demin, Qian Huanyan, Wang Zheng, Yan Xiaoyong. Multicast routing protocol based on energy balance for wireless sensor networks [J]. Sensor and Microsystem. 2010 (11) 